\title{
(2) OPEN ACCESS \\ Application of mydriasis and eye steering in ultrawide field imaging for detecting peripheral retinal lesions in myopic patients
}

\author{
Meiyan Li, 1,2,3,4 Danjuan Yang, 1,2,3,4 Yang Shen, 1,2,3,4 Jianmin Shang, 1,2,3,4 \\ Lingling Niu, ${ }^{1,2,3,4}$ Yongfu Yu, ${ }^{5}$ Xiaoying Wang (D) , 1,2,3,4 Peijun Yao, 1,2,3,4 \\ Xingtao Zhou (D) $1,2,3,4$
}

\begin{abstract}
Additional supplemental material is published online only. To view, please visit the journal online (http://dx.doi. org/10.1136/bjophthalmol2021-319809)
\end{abstract}

${ }^{1}$ Eye Institute and Department of Ophthalmology, Eye \& ENT Hospital of Fudan University, Shanghai, China

${ }^{2} \mathrm{NHC}$ Key Laboratory of Myopia, Fudan University, Shanghai,

China

${ }^{3}$ Shanghai Research Center of Ophthalmology and Optometry, Shanghai, China

${ }^{4}$ Shanghai Engineering

Research Center of Laser and Autostereoscopic 3D for Vision

Care, Shanghai, China

${ }^{5}$ Department of Biostatistics,

School of Public Health of Fudan

University, Shanghai, China

\section{Correspondence to}

Dr Xingtao Zhou, Fudan University, Shanghai, China; doctzhouxingtao@163.comDr Peijun Yao; pjyao@hotmail.com

$\mathrm{ML}$ and DY contributed equally.

Received 8 June 2021

Accepted 16 February 2022

Check for updates

(C) Author(s) (or their employer(s)) 2022. Re-use permitted under CC BY-NC. No commercial re-use. See rights and permissions. Published by BMJ.

To cite: Li M, Yang D, Shen Y, et al. Br J Ophthalmol Epub ahead of print: [please include Day Month Year]. doi:10.1136/

bjophthalmol-2021-319809

\section{ABSTRACT}

Purpose To compare mydriatic and eye-steering ultrawide field imaging (UWFI) with standard nonmydriatic UWFI examination in detecting peripheral retinal lesions in myopic patients.

Methods Cross-sectional, observational study. 220 eyes of 110 myopic patients with known peripheral retinal lesions in at least one eye under Goldmann three mirror contact lens examination were recruited. Non-mydriatic standard and eye-steering UWFI images were taken centrally and with eye-steering technique in upper, lower, nasal and temporal gazes under Optomap UWFI (Daytona, Optos, UK). Mydriatic standard and eye-steering UWFI was captured in central gaze and four different peripheral gazes. Sensitivity of detecting peripheral retinal lesions under different UWFI settings was compared.

Results 141 (64.09\%) eyes were with peripheral retinal lesions. The sensitivity for detecting peripheral lesions from low to high was $41.84 \%$ (95\% Cl 33.62\% to $50.54 \%)$ under non-mydriatic standard UWFI setting, $52.48 \%$ (95\% Cl $44.08 \%$ to $60.75 \%$ ) under mydriatic standard setting, $75.18 \%(95 \% \mathrm{Cl} 67.21 \%$ to $82.06 \%$ ) under non-mydriatic eye-steering setting and $86.52 \%$ (95\% Cl $79.76 \%$ to $91.69 \%$ ) under mydriatic eye-steering setting. Both mydriasis and eye-steering technique increased sensitivity of detecting peripheral lesions with statistical significance $(p<0.001)$. By applying eye-steering technique, sensitivity of detecting lesions located in superior and inferior quadrants witnessed a greater increase compared with other two quadrants $(p<0.05)$. Neither spherical equivalence ( $p>0.05$ ) nor axial length ( $p>0.05$ ) was an independent influence factor for detecting peripheral lesions.

Conclusions Eye-steering technique and mydriasis could both efficiently improve the sensitivity of detecting peripheral retinal lesions in myopic patients. Lesions of superior and inferior quadrants benefited more from eyesteering technique.

\section{INTRODUCTION}

The incidence of myopia has been increasing globally each year ${ }^{1}$ and myopia-related maculopathy has been found to be one of most common causes for blindness. ${ }^{2}$ Nowadays, more than 3 million myopic patients have been seeking for surgical treatment to correct myopia, with nearly half of them were high myopes. ${ }^{34}$ Previous studies showed that high myopia itself, retinal holes or tears, peripheral degenerations like lattice degeneration and planned intraocular surgeries were all independent risk factors for retinal detachment. ${ }^{5-8}$ The detection and photodocumentation of peripheral retinal pathologies, especially those predisposing retinal detachment, were in demand in clinical practice. ${ }^{9}$

Ultrawide field imaging (UWFI) uses ellipsoid mirror to obtain an unprecedented large-angle view of up to $200^{\circ}$ without pupillary mydriasis and have achieved a wide application in diabetic retinopathy, ${ }^{10}$ uveitis, ${ }^{11}$ age-related macular degeneration ${ }^{12}$ and myopia, ${ }^{13}$ etc. It has a profound advantage over traditional retinal examinations like indirect binocular ophthalmoscope, Goldmann three mirror contact lens by providing image-based counselling for patients with peripheral retinal lesions. ${ }^{14}$

By manipulating eye-steering technique, there were reported cases that lesions that missed out in standard gaze could be detected. ${ }^{15} 16$ Sensitivity of detecting peripheral retinal lesions in UWFI would theoretically be elevated under eye-steering setting.

In simulated mydriatic state, model eyes were found to obtain a wider angle view under UWFI. ${ }^{17}$ Mydriasis would possibly benefit patients with small pupils or lesions that situated in far periphery. ${ }^{15}$ However, there has been no reports of UWFI allied with mydriasis and eye-steering technique among patients with peripheral retinal lesions.

The current application of UWFI in clinical scenario was mostly in the non-mydriatic and standard settings without fully using the inherent imaging acquisition software of 'the eye-steering mode' to obtain the possibly maximum visualised retinal area. ${ }^{1018}$ This study aims to investigate the efficacy of UWFI allying mydriasis and eye-steering technique in detecting peripheral retinal lesions in myopic patients.

\section{METHODS}

\section{Study design and participants}

A cross-sectional, observational study: 110 myopic patients were enrolled from November 2019 to July 2020 in Eye and ENT Hospital of Fudan University. The eligibility criteria for this study were: aged $\geq 18$ years, with known peripheral retinal lesions under Goldmann three-mirror contact lens examination in at least one eye. Patients with history of adverse reaction to pupil dilation, inability of eye fixation, 


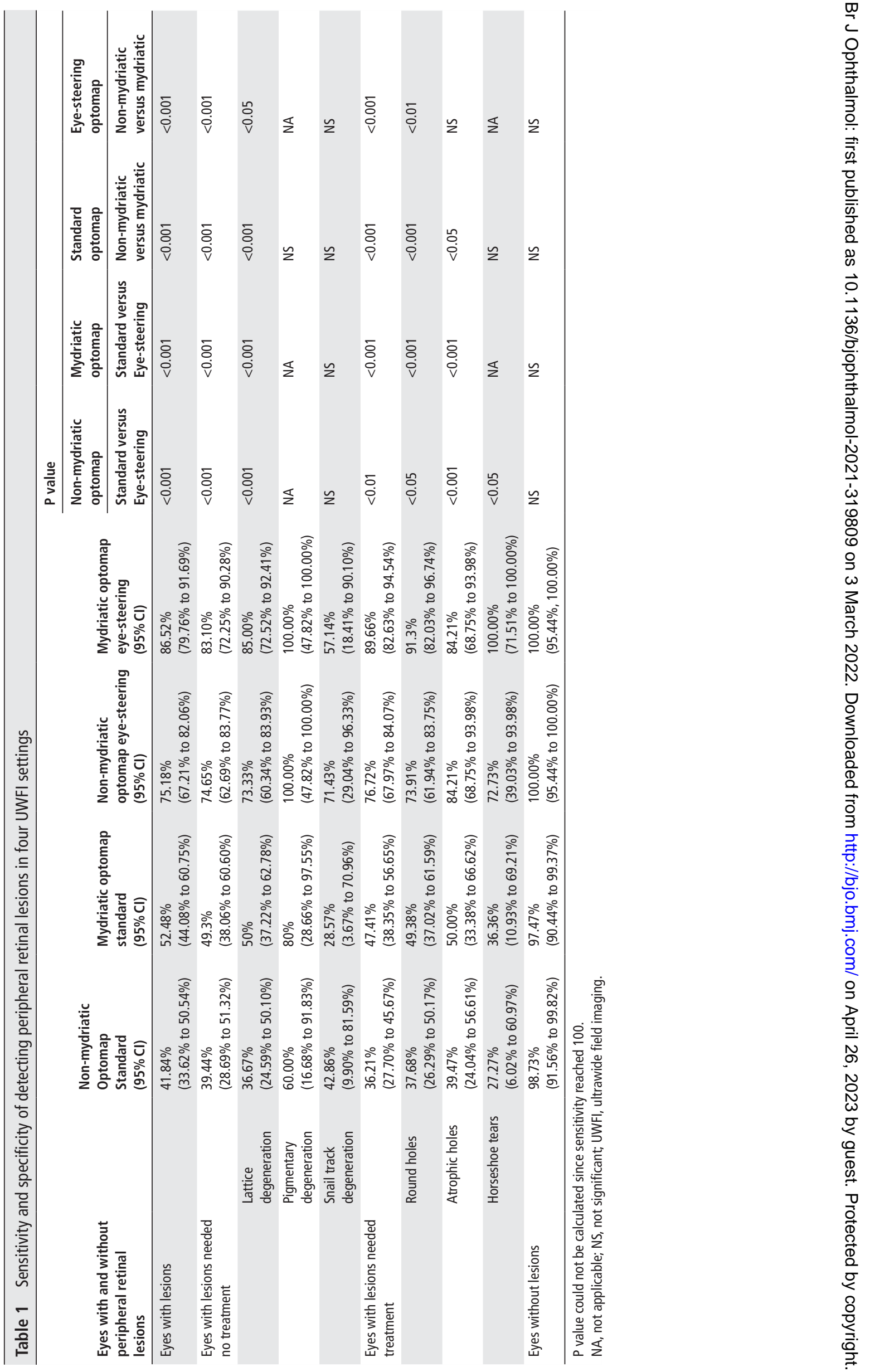




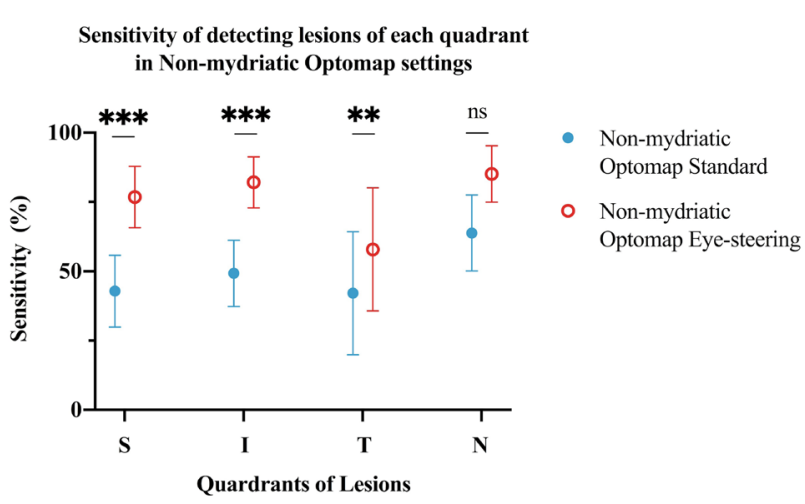

A

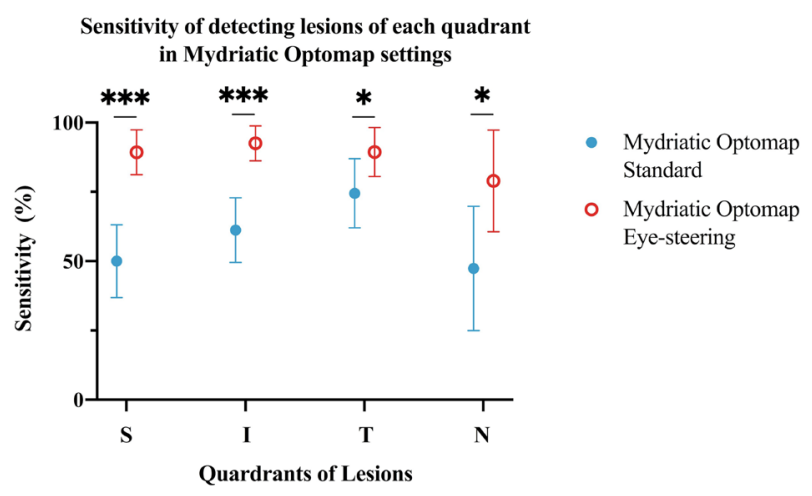

B

Figure 1 Sensitivity of detecting lesions of each quadrant under nonmydriatic (A) and mydriatic (B) eye-steering UWFI. I, inferior; N, nasal; S, superior; T, temporal; UWFI, ultrawide field imaging.ns:not significant; ${ }^{*} \mathrm{P}<0.05 ;{ }^{* *} \mathrm{P}<0.01 ;{ }^{* *} \mathrm{P}<0.001$.

systemic or ocular diseases other than myopia that interfering with image acquisition like cataract, vitreous haemorrhage, etc were excluded from this study.

\begin{tabular}{|c|c|c|c|c|c|c|}
\hline Location & Nasal & Temporal & Superior & Inferior & $\begin{array}{l}\text { Whole } \\
\text { periphery } \\
\text { (detected } \\
\text { proportion\%) }\end{array}$ & $\begin{array}{l}\text { Lesion } \\
\text { no }\end{array}$ \\
\hline $\begin{array}{l}\text { (A) Lesions needed } \\
\text { treatment }\end{array}$ & \multicolumn{6}{|c|}{ Gazes of non-mydriatic UWFI under eye-steering } \\
\hline Nasal & 6 & 0 & 4 & 3 & $9(75.00)$ & 12 \\
\hline Temporal & 1 & 21 & 20 & 13 & $31(86.11)$ & 36 \\
\hline Superior & 3 & 6 & 27 & 4 & $30(76.92)$ & 39 \\
\hline Inferior & 5 & 14 & 4 & 35 & $39(69.64)$ & 56 \\
\hline Total & 15 & 41 & 55 & 55 & $109(76.22)$ & 143 \\
\hline $\begin{array}{l}\text { (B) All recorded } \\
\text { lesions }\end{array}$ & \multicolumn{6}{|c|}{ Gazes of mydriatic UWFI under eye-steering } \\
\hline Nasal & 9 & 2 & 11 & 9 & $17(80.95)$ & 21 \\
\hline Temporal & 4 & 45 & 43 & 24 & $60(89.55)$ & 67 \\
\hline Superior & 10 & 19 & 61 & 12 & $66(90.41)$ & 73 \\
\hline Inferior & 13 & 32 & 15 & 76 & $84(90.32)$ & 93 \\
\hline Total & 36 & 98 & 130 & 121 & $227(89.37)$ & 254 \\
\hline
\end{tabular}

(B) All recorded lesions under different gazes of mydriatic UWFI.

UNFI, ultrawide field imaging.
In this study, 220 eyes of 110 myopic patients (75 female, 35 male) with a mean age of $28.08 \pm 6.37$ (ranging from 18 to 50 ) were included in this study. The spherical equivalence of study eyes averaged $-8.76 \pm 4.24$ dioptres, ranging from -28.88 to -1.25 dioptres. The axial length of study eyes averaged $27.28 \pm 1.75 \mathrm{~mm}$, ranging from 24.52 to $34.85 \mathrm{~mm}$.

\section{Image acquisition}

All the images were taken under Optomap Panoramic Daytona device (Daytona, Optos, UK) in pseudocoloured pattern obtained by dual lasers at $532 \mathrm{~nm}$ and $633 \mathrm{~nm}$. A masked experienced ophthalmologist performed the UWFI in a dark room without knowing the location and type of retinal pathologies and other ophthalmic findings. Patients were guided to look inside the green dot to get non-mydriatic UWFI standard images with patients' eyes centred on the fovea. In the eye-steering settings, patients were guided to look at the red dot inside the UWFI device with a slight shifting of the gaze in the superior, inferior, temporal or nasal direction in turn to get the peripheral view of fundus.

Tropicamide Phenylephrine Eye-Drops $(0.5 \%$ tropicamide and $0.5 \%$ epinephrine, Santen Pharmaceutical, Japan) were applied in subjects' eyes every $10 \mathrm{~min}$ for three times to dilate the pupils. Pupils were regarded fully dilated when they were completely unresponsive to pen light. Mydriatic UWFI standard and eye-steering images were taken the same as in the nonmydriatic state.

In four UWFI settings, any image with the macula and the optic disc obscured or peripheral lid artefacts covering more than $50 \%$ mid-peripheral area was defined insufficient image acquisition and would be retaken instantly to get a qualified one.

\section{Grading protocol}

Images were all reviewed under Optos V.2 Vantage Pro Review V.2.8.0 software (Optos, Dunfermline, UK). Grading of each image was based on the pseudocoloured imaging. All the images were graded independently by two ophthalmologists with types and locations of the all lesions recorded. Both reviewers were masked to other ophthalmic findings of enrolled patients. One of the ophthalmologists regraded the same images after 1-month wash-out interval for assessing interobserver agreement.

Images that the two graders had diverged opinions on were reassessed by another specialist using localised magnification, 3D stereo projection or combing red-free and green-free channels to determine the final results. The final assessment under UFWI was compared with dilated fundus examination with Goldmann three mirror contact lens.

The fundus was divided according to clock hours and four quadrants as following: superior quadrant (10:30-1:30 clock hours), inferior quadrant (from 4:30 to 7:30 clock hours), temporal quadrant $(7: 30-10: 30$ for right eye; $1: 30-4: 30$ for left eye), nasal quadrant (1:30-4:30 for right eye; 7:30-10:30 for left eye).

\section{Data collection and analysis}

Categorical variables were summarised by frequencies and percentages. Continuous variables were summarised by mean \pm SD. A sample size at least 62 eyes with peripheral retinal lesions was calculated with an estimated sensitivity of $80 \%$ and precision of estimate of $0.1 .{ }^{19}{ }^{20}$ Sensitivity was defined as true positive/(true positive + false negative). Specificity was defined as true negative/(true negative + false positive). McNemar paired test was applied to compare sensitivity and specificity in different 

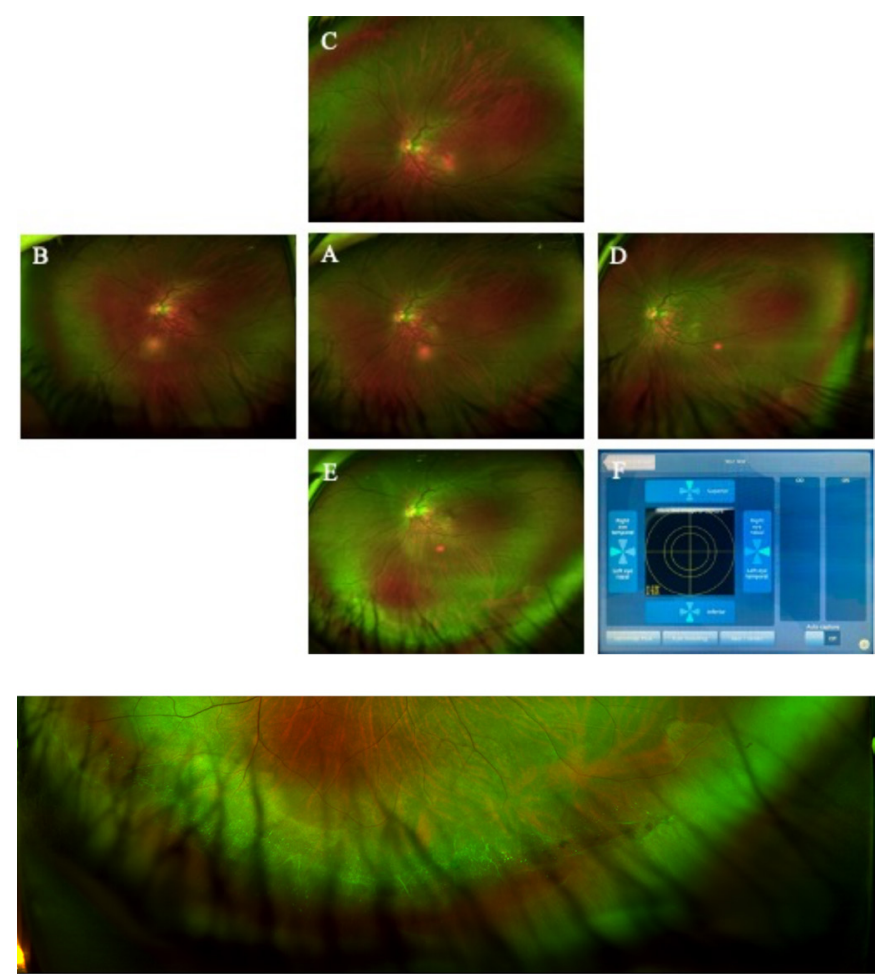

Figure 2 Example of peripheral retinal lesions detected in corresponding gaze under eye-steering UWFI. (A) Standard gaze, (B) nasal eye-steering, (C) superior eye-steering, (D) temporal eyesteering, (E) inferior eye-steering. lattice degeneration and an atrophic hole (white arrow) were only observed in inferior gaze and missed out in standard and all other three gazes. (F) Interface of the eye-steering software. UWFI, ultrawide field imaging.

UWFI settings. Cochran-Mantel-Haenszel test was used to further explore the difference of detecting peripheral lesions in low-tomoderate (SE $\geq-6.00 \mathrm{D})$, high $(-10.00 \mathrm{D}<\mathrm{SE}<-6.00 \mathrm{D})$ and ultrahigh (SE $<-10.00 \mathrm{D})$ myopic subjects. To avoid the impact of the fellow eye in this study, generalised estimating equations were applied to avoid the influence of the contralateral eye with the fellow eye as covariate. Statistical analyses were performed with SAS V.9.4 (SAS Institute). A p $<0.05$ was considered statistically significant.

\section{RESULTS}

\section{Frequency and distribution of peripheral retinal lesions}

$64.09 \%(141 / 220)$ eyes were with peripheral retinal lesions while 35.91\% (79/220) were without peripheral pathologies. Of all eyes with peripheral lesions, 116 (52.73\%) eyes had peripheral retinal holes or tears. Sixty-nine (31.36\%) eyes were with round holes followed by $38(17.27 \%)$ eyes with atrophic holes and $11(5.00 \%)$ eyes with horseshoe tears. Of all eyes with peripheral retinal degenerations, 60 (27.27\%) eyes were with lattice degeneration, followed by 7 (3.18\%) eyes with snail track degeneration and 5 (2.27\%) eyes with pigmentary degeneration. 25.91\% (57/220) eyes had more than one type of peripheral pathology and $29.54 \%(65 / 220)$ eyes had peripheral lesions in more than one quadrant. The frequency of peripheral retinal lesions distributed in each semi meridian numbered according to clock hours was detailed in online supplemental figure 1 .
Lesions detected by UWFI in four settings: non-mydriatic UWFI standard, non-mydriatic UWFI eye steering, mydriatic UWFI standard and mydriatic UWFI eye steering

All enrolled eyes completed UWFI under four settings. The interobserver kappa value was $0.84 \pm 0.03$ for UWFI reading between the two graders. The interobserver kappa value was $0.91 \pm 0.01$ before and after 1 -month wash-out.

Sensitivity for detecting each specific lesion was summarised in table 1 . Sensitivity was of no statistical significance between different subtypes of retinal holes or between peripheral retinal holes and degenerations $(\mathrm{p}>0.05)$.

By allying eye-steering technique, sensitivity of detecting peripheral retinal lesions increased by $33.33 \%$ (95\% CI $25.31 \%$ to $41.26 \%$ ) and $34.04 \%$ (95\% CI $26.22 \%$ to $41.86 \%$ ) in nonmydriatic and mydriatic state. Mydriasis increased the sensitivity of detecting peripheral retinal lesions by $10.64 \%(95 \% \mathrm{CI}$ $4.21 \%$ to $17.07 \%$ ) and $11.35 \%$ (95\% CI $26.22 \%$ to $41.86 \%$ ) in standard and eye-steering gazes. Both mydriasis and eyesteering technique increased sensitivity of detecting peripheral lesions with statistical significance $(\mathrm{p}<0.001)$. The sensitivity of detecting peripheral degenerations were of no difference compared with that of detecting peripheral retinal holes under all four settings of UWFI ( $p>0.05)$. Specificity of detecting peripheral retinal lesions remained unchanged with either mydriasis or eye-steering technique $(\mathrm{p}<0.001)$.

\section{Comparison of detecting lesions of each quadrant under UWFI}

By applying eye-steering technique, sensitivity of detecting lesions located in superior quadrant underwent maximal increase compared with that of all other three quadrants $(\mathrm{p}<0.05)$. Lesions of superior quadrant increased from $42.86 \%$ (95\% CI $29.90 \%$ to $55.82 \%$ ) to $76.79 \%$ (95\% CI $65.73 \%$ to $87.84 \%$ ) under nonmydriatic state $(\mathrm{p}<0.001)$ and from $50.00 \%(95 \%$ CI $36.90 \%$ to $63.10 \%)$ to $89.29 \%$ (95\% CI $81.18 \%$ to $97.39 \%)$ under mydriatic state $(\mathrm{p}<0.001)$. Lesions of inferior quadrant also witnessed the increase of sensitivity under both non-mydriatic (from $49.25 \%$ to $82.09 \%$ ) and mydriatic (from $61.19 \%$ to $92.54 \%$ ) settings $(\mathrm{p}<0.001)$. Detailed sensitivity of each quadrant was shown in figure 1.

\section{Peripheral lesions detected with eye-steering technique in four gazes}

Distribution of detected peripheral retinal lesions in four gazes conformed to a 'diagonal principle', namely lesions of one specific quadrant could be most likely detected in the corresponding gaze. Numbers of peripheral lesions and those in need of treatment detected in different gazes of non-mydriatic and mydriatic UWFI were available in table $2 \mathrm{~A}, \mathrm{~B}$, respectively. Lesions that could be detected in the corresponding gaze only accounted for $79.38 \%(154 / 194)$ and $84.14 \%(191 / 227)$ of all detectable lesions under non-mydriatic and mydriatic settings. With the help of images from other three gazes, the detection rate increased with statistical significance $(\mathrm{p}<0.001)$ in both non-mydriatic (increased by $15.75 \%$ ) and mydriatic circumstances (increased by 14.17\%). An example of peripheral retinal lesions that missed in the standard setting but detected in the corresponding gaze with eye steering was shown in figure 2 .

\section{Detection of peripheral retinal lesions of different myopia severity}

The sensitivity of detecting peripheral retinal lesions in low-tomoderate, high and ultrahigh myopic subjects were 38.71\% (95\% 


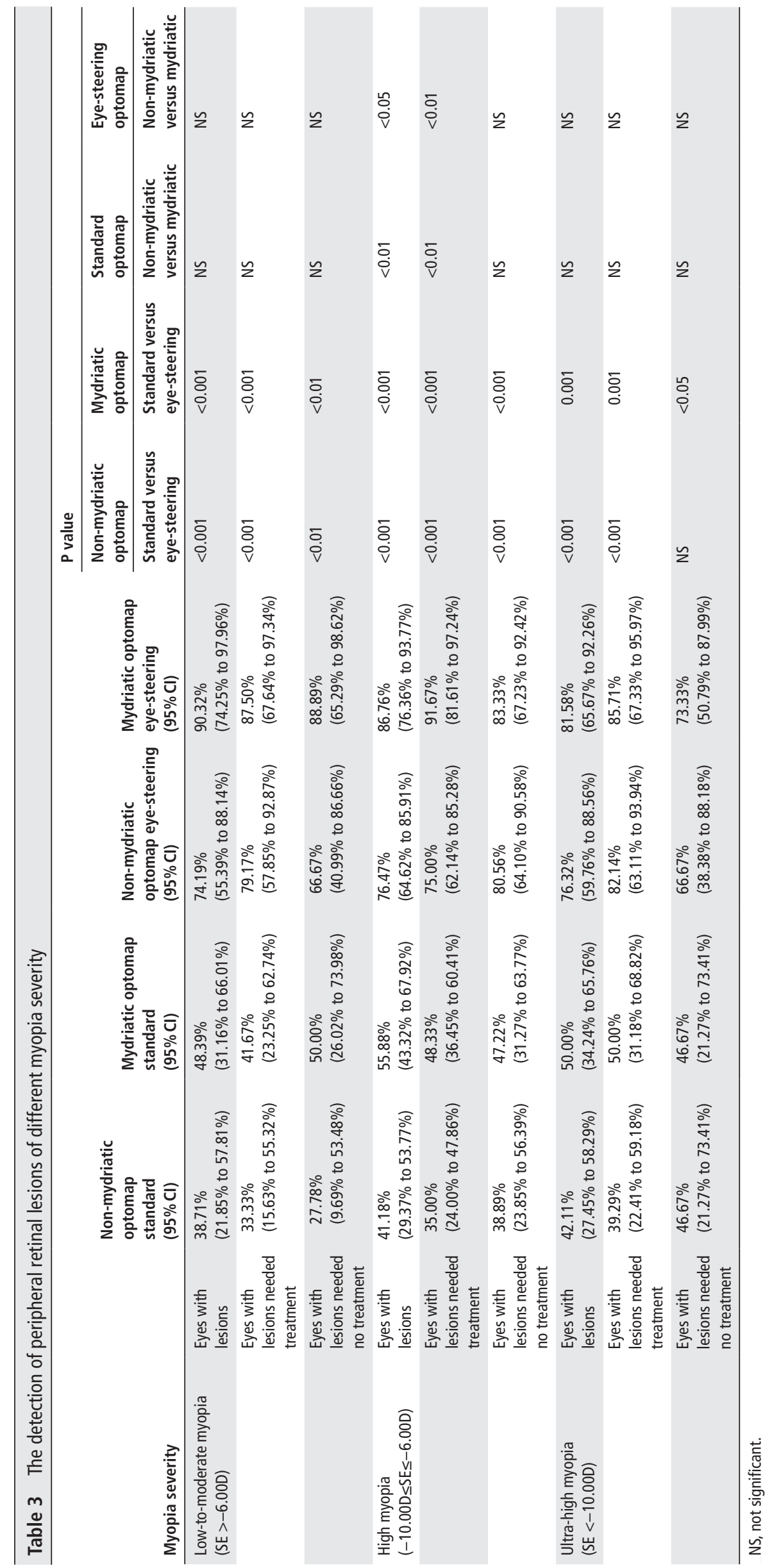


CI $21.85 \%$ to $57.81 \%$ ), $41.18 \%$ (95\% CI $29.37 \%$ to $53.77 \%$ ) and $42.11 \%$ (95\% CI $27.45 \%$ to $58.29 \%$ ) under non-mydriatic standard UWFI and increased to $90.32 \%$ (95\% CI $74.25 \%$ to $97.96 \%$ ), $86.76 \%$ (95\% CI $76.36 \%$ to $93.77 \%$ ) and $81.58 \%$ (95\% CI $65.67 \%$ to $92.26 \%$ ) with mydriasis and eye-steering technique applied. No statistical difference was observed in each UWFI setting between the three groups. Detection of peripheral retinal lesions of different myopia severity was summarised in table 3. Since spherical equivalence and axial length were strongly correlated $\left(\mathrm{R}^{2}=0.71\right)$, the detection of peripheral retinal lesions of different axial length group was detailed in online supplemental table 1 . Neither spherical equivalence $(\mathrm{p}>0.05)$ nor axial length $(p>0.05)$ was an independent influence factor for detecting peripheral lesions after adjusting for age and sex in each UWFI setting.

\section{DISCUSSION}

UWFI has long been applied in clinic practice for assessing and photo-documenting vitreoretinal pathologies, with an advantage of visualising the peripheral area. ${ }^{21-23}$ It was not yet clear that whether or not mydriasis or eye-steering technique could increase the sensitivity of detecting peripheral retinal lesions in clinical practice.

In this study, mydriasis helped increase the sensitivity in standard gaze position by around $10 \%$ for peripheral retinal lesions and also for retinal holes and degenerations. It is in consistency with the results from Oishi et al in model eyes which proved that eyes with an $8 \mathrm{~mm}$ pupil had a $10^{\circ}$ increase in visualised retinal area in each direction compared with that of pupils with a diameter of $2 \mathrm{~mm}^{17}$

Eye-steering technique hugely improved the sensitivity of detecting peripheral lesions by more than $30 \%$, not only in the non-mydriatic condition but also in the mydriatic circumstance. Gupta et al found that by applying eye-steering technique in UWFI, $34.5 \%$ of retinal area could be revisualised. ${ }^{24}$ Mydriatic UWFI combined with eye-steering technique could achieve the highest sensitivity up to $86.52 \%$ in this study. It could be comparable to the sensitivity of 5 -gaze position (ahead, superior, inferior, right, left) in the latest version of UWFI Optos California (89.2\%). ${ }^{20}$ And it much exceeded the sensitivity of nonmydriatic standard UWFI reported by previous studies, ranging from $33 \%$ to $74 \% .^{25-28}$

Peripheral retinal lesions of superior and inferior quadrants were reported more susceptible to be missed out in clinic practice. ${ }^{25}{ }^{29}$ For UWFI, nasal and temporal quadrants exposed much more retina area than superior and inferior ones under simulated non-mydriatic and mydriatic pupils. ${ }^{30} 31$ Chen et al also proved in patients' eyes the asymmetry of captured retinal area for horizontal and vertical directions. ${ }^{32}$ Our study proved that lesions situated in superior and inferior quadrants benefited more from eye-steering technique, which implied an approach of overcoming the disadvantage of UWFI designing that using an elliptical mirror. ${ }^{17}$

In this study, a detectable lesion under UWFI could not be $100 \%$ detected in the corresponding gaze, even under the mydriatic condition. One reason is that the detected retinal area in four corresponding gazes was not strictly the same as the divided four quadrants. Another reason is, as stated above, the disparity of captured retinal area under UWFI.

A thorough and careful preoperative fundus examination was necessary for myopic patients, as $88.2 \%$ postoperative peripheral retinal breaks were adjacent to previous detected lesions. ${ }^{33}$ Up to now, the effects of surgical interventions in eyes with asymptomatic retinal holes has not yet been verified. ${ }^{34}$ The interventional strategy in this study was that all peripheral retinal holes, regardless of subtypes, were all treated with laser photocoagulation, peripheral retinal degenerations were suggested to be followed up.

Lesions that needed treatment were more prioritised by refractive surgeons. The results of this study showed that peripheral lesions that needed prophylactic treatment were of no difference in detection compared with that needed no treatment under all four settings of UWFI.

Neither axial length nor spherical equivalence influenced the sensitivity of detecting peripheral retinal lesions in each UWFI setting, which actually confirmed to the clinical experience. And previous studies have already proved that high myopia and long axial length had more impact on the sharpness of peripheral retina rather the visualised retinal area. ${ }^{243}$

Limitations of this study include, first, this was a cross-sectional study without follow-up image data. Second, limited number of specific peripheral retinal lesions reduced the power of analysing difference between the detection of different lesions. Third, the inherent distortion and loss of image sharpness of the peripheral retina could not be dismissed in the process of flattening of the three-dimensional image of the eyeball to generate a twodimensional fundus photograph.

In summary, mydriasis and eye-steering technique could both efficiently improve the sensitivity of detecting peripheral retinal lesions in myopic patients. Lesions of superior and inferior quadrants benefited more from eye-steering technique.

Contributors ML and DY: Conceptualisation, data collection, manuscript drafting, critical revision and statistical analysis. YS, JS, LN and XW: Data collection and manuscript drafting. YY: Data collection and analyzing. PY and XZ: Conceptualisation, critical revision of manuscript, funding and supervision. All authors approved the final submission of this manuscript. XZ is responsible for the overall content as guarantor.

Funding (1) National Natural Science Foundation of China (Grant No. 81770955). (2) Joint research project of new frontier technology in municipal hospitals (SHDC12018103). (3) Project of Shanghai Science and Technology (Grant No.20410710100). (4) Clinical Research Plan of SHDC (SHDC2020CR1043B). (5) Project of Shanghai Xuhui District Science and Technology (2020-015). (6) Shanghai Rising-Star Programme (21QA1401500).

Competing interests None declared.

Patient consent for publication Not applicable.

Ethics approval This study was approved by Ethical Committee of Eye and ENT Hospital of Fudan University Review Board (2016038) and the study was conducted in accordance with the tenets of the Declaration of Helsinki.

Provenance and peer review Not commissioned; externally peer reviewed.

Data availability statement Data are available on reasonable request. Availability of data and materials: Available from the corresponding author on reasonable request. Email: doctzhouxingtao@163.com.

Supplemental material This content has been supplied by the author(s). It has not been vetted by BMJ Publishing Group Limited (BMJ) and may not have been peer-reviewed. Any opinions or recommendations discussed are solely those of the author(s) and are not endorsed by BMJ. BMJ disclaims all liability and responsibility arising from any reliance placed on the content. Where the content includes any translated material, BMJ does not warrant the accuracy and reliability of the translations (including but not limited to local regulations, clinical guidelines, terminology, drug names and drug dosages), and is not responsible for any error and/or omissions arising from translation and adaptation or otherwise.

Open access This is an open access article distributed in accordance with the Creative Commons Attribution Non Commercial (CC BY-NC 4.0) license, which permits others to distribute, remix, adapt, build upon this work non-commercially, and license their derivative works on different terms, provided the original work is properly cited, appropriate credit is given, any changes made indicated, and the use is non-commercial. See: http://creativecommons.org/licenses/by-nc/4.0/.

\section{ORCID iDs}

Xiaoying Wang http://orcid.org/0000-0001-5419-6318 
Xingtao Zhou http://orcid.org/0000-0002-3465-1579

\section{REFERENCES}

1 Morgan IG, French AN, Ashby RS, et al. The epidemics of myopia: aetiology and prevention. Prog Retin Eye Res 2018;62:134-49.

2 Iwase A, Araie M, Tomidokoro A, et al. Prevalence and causes of low vision and blindness in a Japanese adult population: the Tajimi study. Ophthalmology 2006:113:1354-62.

3 Wang Y, Ma J. Future developments in SMILE: higher degree of myopia and hyperopia. Asia Pac J Ophthalmol 2019;8:412-6.

4 Wang X, Zhou X. Update on treating high myopia with implantable collamer lenses. Asia Pac J Ophthalmol 2016;5:445-9.

5 Ohno-Matsui K, Lai TYY, Lai C-C, et al. Updates of pathologic myopia. Prog Retin Eye Res 2016;52:156-87.

6 Lewis H. Peripheral retinal degenerations and the risk of retinal detachment. Am J Ophthalmol 2003;136:155-60.

7 Risk factors for idiopathic rhegmatogenous retinal detachment. the eye disease casecontrol study Group. Am J Epidemiol 1993;137:749-57.

8 Powe NR, Schein OD, Gieser SC, et al. Synthesis of the literature on visual acuity and complications following cataract extraction with intraocular lens implantation. cataract patient outcome research team. Arch Ophthalmol 1994;112:239-52.

9 Kumar J, Kohli P, Babu N, et al. Comparison of two ultra-widefield imaging for detecting peripheral retinal breaks requiring treatment. Graefes Arch Clin Exp Ophthalmol 2021;259:1427-34.

10 Szeto SKH, Wong R, Lok J, et al. Non-mydriatic ultrawide field scanning laser ophthalmoscopy compared with dilated fundal examination for assessment of diabetic retinopathy and diabetic macular oedema in Chinese individuals with diabetes mellitus. Br J Ophthalmol 2019;103:1327-31.

11 Campbell JP, Leder HA, Sepah YJ, et al. Wide-Field retinal imaging in the management of noninfectious posterior uveitis. Am J Ophthalmol 2012;154:908-11.

12 Guduru A, Fleischman D, Shin S, et al. Ultra-Widefield fundus autofluorescence in age-related macular degeneration. PLoS One 2017;12:e0177207.

13 Liu L, Wang F, Xu D, et al. The application of wide-field laser ophthalmoscopy in fundus examination before myopic refractive surgery. BMC Ophthalmol 2017;17:250

14 Sharma A, Dongre P, Bellala K, et al. Role of retinal image-based counseling in the treatment of peripheral retinal lesions. Eye 2019;33:161-3.

15 Jones WL. Limitations of the panoramic 200 Optomap. Optom Vis Sci 2004:81:165-6.

16 Nagiel A, Lalane RA, Sadda SR, et al. Ultra-Widefield fundus imaging. Retina 2016:36:660-78.

17 Oishi A, Hidaka J, Yoshimura N. Quantification of the image obtained with a wide-field scanning ophthalmoscope. Invest Ophthalmol Vis Sci 2014;55:2424-31.

18 Hirano T, Imai A, Kasamatsu $\mathrm{H}$, et al. Assessment of diabetic retinopathy using two ultra-wide-field fundus imaging systems, the Clarus ${ }^{\circledR}$ and Optos ${ }^{\mathrm{TM}}$ systems. BMC Ophthalmol 2018:18:332.
19 Hajian-Tilaki K. Sample size estimation in diagnostic test studies of biomedical informatics. J Biomed Inform 2014:48:193-204.

20 Fogliato G, Borrelli E, Iuliano L, et al. Comparison between ultra-widefield Pseudocolor imaging and indirect ophthalmoscopy in the detection of peripheral retinal lesions. Ophthalmic Surg Lasers Imaging Retina 2019;50:544-9.

21 Lei B, Zhou M, Wang Z, et al. Ultra-wide-field fundus imaging of acute retinal necrosis: clinical characteristics and visual significance. Eye 2020;34:864-72.

22 Son G, Kim YJ, Sung YS, et al. Analysis of quantitative correlations between microaneurysm, ischaemic index and new vessels in ultrawide-field fluorescein angiography images using automated software. Br J Ophthalmol 2019;103:bjophthalmol-2018-313596-64.

23 Quinn N, Csincsik L, Flynn E, et al. The clinical relevance of visualising the peripheral retina. Prog Retin Eye Res 2019;68:83-109.

24 Gupta A, El-Rami H, Barham R, et al. Effect of phase-plate adjustment on retinal image sharpness and visible retinal area on ultrawide field imaging. Eye 2019:33:587-91.

25 Yang D, Li M, Wei R, et al. Optomap ultrawide field imaging for detecting peripheral retinal lesions in 1725 high myopic eyes before implantable collamer lens surgery. Clin Exp Ophthalmol 2020;48:895-902.

26 Mackenzie PJ, Russell M, Ma PE, et al. Sensitivity and specificity of the optos optomap for detecting peripheral retinal lesions. Retina 2007;27:1119-24.

27 Khandhadia S, Madhusudhana KC, Kostakou A, et al. Use of Optomap for retinal screening within an eye casualty setting. Br J Ophthalmol 2009;93:52-5.

28 Darwish DY, Patel SN, Gao Y, et al. Diagnostic accuracy and reliability of retinal pathology using the Forus 3nethra fundus camera compared to ultra wide-field imaging. Eye 2019;33:856-7.

29 Kornberg DL, Klufas MA, Yannuzzi NA, et al. Clinical utility of ultra-widefield imaging with the Optos Optomap compared with indirect ophthalmoscopy in the setting of non-traumatic rhegmatogenous retinal detachment. Semin Ophthalmol 2016;31:505-12.

30 Witmer MT, Parlitsis G, Patel S, et al. Comparison of ultra-widefield fluorescein angiography with the Heidelberg Spectralis $(\circledR)$ noncontact ultra-widefield module versus the Optos(@) Optomap(®). Clin Ophthalmol 2013;7:389-94.

31 Kato $Y$, Inoue $M$, Hirakata A. Quantitative comparisons of ultra-widefield images of model eye obtained with Optos ${ }^{\circledR} 200 T_{x}$ and Optos ${ }^{\circledR}$ California. BMC Ophthalmol 2019;19:115.

32 Chen A, Dang S, Chung MM. Quantitative comparison of fundus images by two UltraWide field fundus cameras. Ophthalmology Retina 2020.

33 Chan CK, Tarasewicz DG, Lin SG. Relation of pre-LASIK and post-LASIK retinal lesions and retinal examination for LASIK eyes. Br J Ophthalmo/ 2005;89:299-301.

34 Wilkinson C. Interventions for asymptomatic retinal breaks and lattice degeneration for preventing retinal detachment. Cochrane Database Syst Rev 2001;3:CD003170.

35 Lim S-H, Jeong S, Ahn JH, et al. Effects of refractive power on quantification using ultra-widefield retinal imaging. BMC Ophthalmol 2021:21:141. 